\title{
Switchable diffractive optics using patterned PEDOT:PSS based electrochromic thin-films
}

\author{
Zefram D. Marks ${ }^{1}$, David Glugla ${ }^{2}$, Jacob T. Friedlein ${ }^{2}$, Sean E. Shaheen ${ }^{2}$, Robert R. McLeod ${ }^{2}$, Malik Y. Kahook ${ }^{1}$ \\ and Devatha P. Nair ${ }^{1}$ \\ ${ }^{1}$ University of Colorado Anschutz Medical Campus, Department of Ophthalmology, Aurora, Colorado, USA \\ ${ }^{2}$ University of Colorado Boulder, Department of Electrical Engineering, Boulder, Colorado, USA
}

\begin{abstract}
We demonstrate switchable, thin film diffractive optical gratings and Fresnel zone plate lenses fabricated using a micro-patterned electrochromic polymer and gel electrolyte. Electrochemically switching the conductive polymer PEDOT:PSS causes the patterned layer to change between a low-absorption to high-absorption state, acting as an amplitude diffractive optical element. The switchable lens and gratings were fabricated using a lithographically patterned electrochromic polymer, a gel electrolyte, and an ITO coated glass substrate. Within an applied voltage of $-1 \mathrm{~V}$ to $1 \mathrm{~V}$, the diffraction efficiency of the switchable lens can be varied 4.1-fold between the 'on' and 'off' states. Due to their low actuation voltage and biocompatibility, electrochemically actuated diffractive optics have potential applications in low power and implantable biomedical devices.
\end{abstract}

\section{Introduction}

Organic and inorganic electrochromic (EC) materials have been used in electrochromic displays and attenuators for over four decades primarily due to ease of processing and cost [1-9]. EC materials reversibly change their absorption spectrum in response to a voltage-driven redox reaction. This effect has been implemented in a variety of devices, including switchable transparency windows ("smart glass") and displays [1,3,10,11]. The electrochromic effect is characteristically observed in response to an applied voltage as an ion exchange between the EC material and an electrolyte which changes the oxidation state of the EC material. The result is a voltage driven change in color of the material, as well as additional material property changes such as conductivity. EC materials include inorganic metal oxides such as $\mathrm{WO}_{3}$, molecular pigments such as viologens and iron ferrocyanide (Prussian Blue) [1,4], and organic conductive polymers such as poly(3,4-ethylene-dioxythiophene) doped with poly(styrene-sulfonate) (PEDOT:PSS) [10,12,13]. Among conductive polymers, PEDOT:PSS is used extensively in the field of organic electronics and EC devices due to its chemical stability, flexibility, and relatively high conductivity of $>1000 \mathrm{~S} / \mathrm{cm}$ [14-17]. In the case of PEDOT:PSS, the electrochemical redox reaction will dope (oxidize) or dedope (reduce) the material thereby changing its conductivity and shifting its absorption spectrum from the infrared into visible wavelengths, which is observed as a darkening of the material. This shift can be exploited in applications that range from electrochemical displays to transistors [18,19]. EC materials such as PEDOT:PSS have intrinsic advantages over other thin-film optical materials such as liquid crystals (LCs), including low voltage DC actuation, ease of processing on flexible substrates, compatibility with both aqueous and organic electrolytes, and demonstrated biocompatibility [20-24]. The explicitly ionic electrochemical actuation mechanism of PEDOT:PSS also enables the materials to interface seamlessly with physiological environments [21,25,26]. Micro-patterned EC devices using amplitude diffraction grating structures have previously been used in EC devices to yield improved coloration efficiency [12,27,28], and recently a diffractive EC device was demonstrated using a 3D nanostructured conjugated polymer EC material, including a Fresnel zone-plate (FZP) lens [29]. However, characterization of the diffraction efficiency of such devices have not been perform, nor has such a system been demonstrated using simple and well understood EC systems such as PEDOT:PSS.

Using diffractive optics, elements such as Fresnel lenses can be made using thin, planar patterns that focus light similar to traditional refractive lenses but have the added advantage of utilizing thin substrates to attain similar optical properties. Diffractive optics use spatially varying amplitude or phase modulation to interfere light and control the spatial distribution of the transmitted light beam. For example, an amplitude FZP lens can be generated from a structure of concentric rings of alternately low and high absorption segments with varying pitch without the volume and mass of material that would be needed to manufacture a similar refractive lens from conventional dielectric material. FZP micro-lens arrays on thin, flexible substrates have been used for wide-field 
imaging in bio-inspired compound eyes [30]. Fixed FZP lenses patterned on top of conventional refractive lenses have been shown to perform well as multifocal intraocular lenses (IOLs) for post-cataract surgery prostheses [31,32]. However, all of the FZP lenses in these studies feature fixed focal lengths and the lens power cannot be altered once fabricated. The ability to dynamically alter the focal length of an optical device by switching a diffractive optical element 'on' or 'off' using an external electrical signal would have significant applications for a range of optical devices such as lenslet arrays and switchable focus optics, including biomedical ophthalmic devices such as electrically switchable focus IOLs [33,34]. By combining the advantages of diffractive optics with active EC materials, a platform in which switchable diffractive optics with the ability to dynamically alter the focus of a lens can be developed. Patterned EC layers can be combined with traditional refractive optics to enable a device to repeatedly and reversibly switch the focal length of a lens in response to an electrical signal.

Although switchable diffractive elements for ophthalmic devices using phase modulating liquid crystals have been studied [33,34], the long-term biocompatibility of LCs along with the need to encapsulate the LCs so as to protect the immediate environment around the devices remains a concern [35]. Unlike LC materials that are generally incompatible with biological systems, PEDOT:PSS has a proven history of biocompatibility and devices made from PEDOT:PSS EC materials have been studied as in vivo biomedical devices [21-23,25,26,3638]. In addition, LC based optical device might require complex voltage modulations and difficult processing conditions, while EC diffractive optics can be actuated using a low-voltage DC signal and are easily solution processed $[1,2,4,13]$. The ability to switch on-and-off a thin-film lens with a low DC voltage can be used to make varifocal contact and IOL devices, with the power supplied using a RF coil and rectifier, or a small onboard power source. A detailed study and characterization of the full potential of active diffractive optics (including diffraction efficiency) using biocompatible EC materials in aqueous electrolytes has been limited todate

In this work, we fabricate and characterize a PEDOT:PSS-based switchable opto-electrochemical device (OECD) that consists of an amplitude modulated diffractive optical elements, including FZP lenses and diffraction grating structures, in which the diffraction efficiency can be controlled as a function of the applied voltage. By utilizing the electrochromism of PEDOT:PSS, a FZP lens can be switched between a nearly uniform low absorption (oxidized) 'off' at state with infinite focal length, to a pattern of alternating low and high absorption (reduced) rings in the 'on' state with a finite focal length, as shown in Figure 1a. The OECDs were fabricated by micro-patterning a spin-coated EC layer of PEDOT:PSS on an ITO-coated glass substrate using conventional lithographic patterning. FZP lenses and amplitude diffraction gratings devices of different sizes were made to characterize the operation and diffraction efficiency. We show that the maximum intensity in the focus of a FZP lens can be changed by a factor of 8.2 with a voltage swing of $2 \mathrm{~V}$, and a diffraction efficiency of up to $0.72 \%$. We also examine the ultimate potential diffraction efficiency of OECDs and their potential as ophthalmic biomedical devices. 

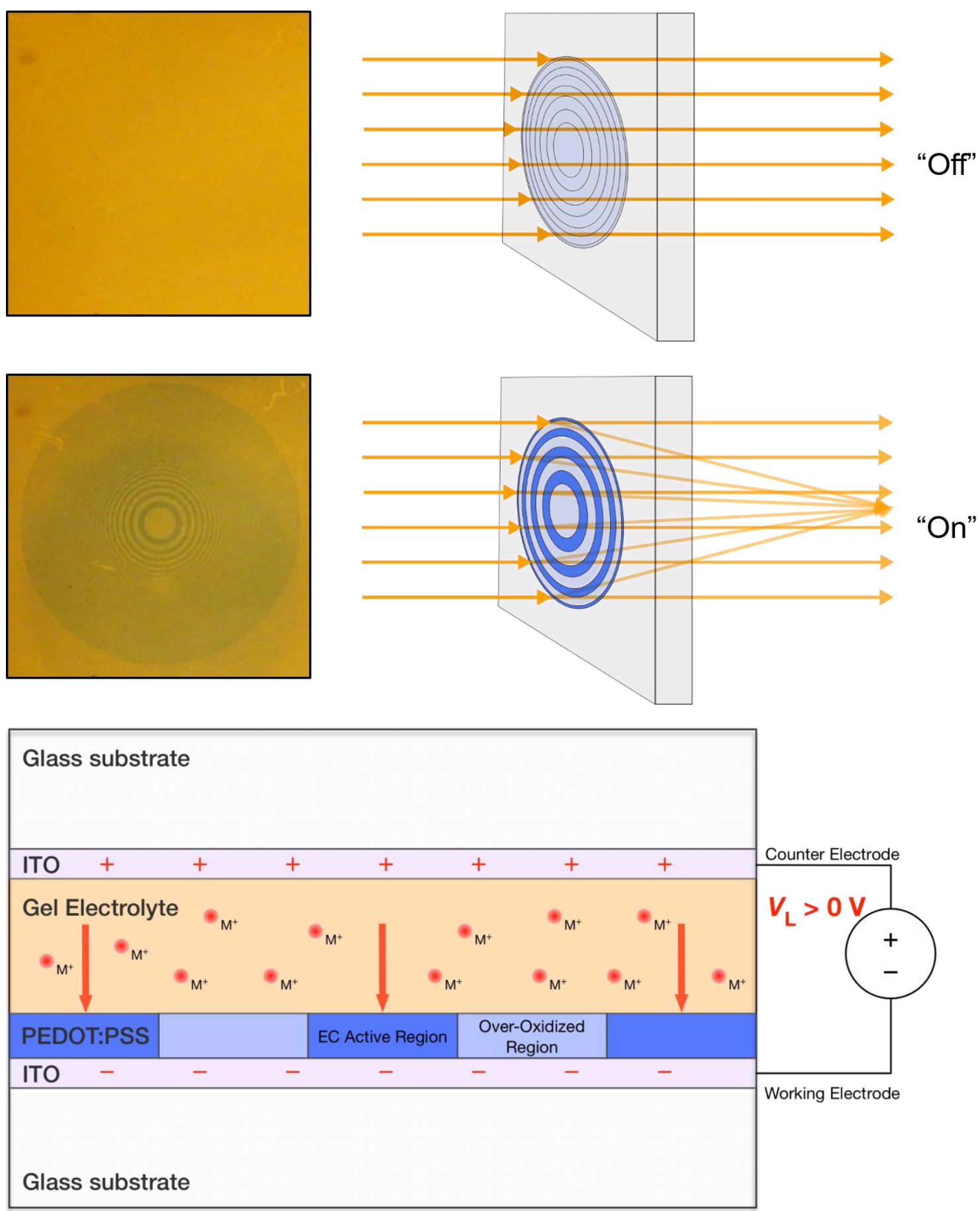

Figure 1: a) Schematic of a switchable FZP OECD and device. When the OECD is 'off' there is minimal contrast between the electrochromically active and over-oxidized inactive regions of the substrate resulting in very low diffraction efficiency and thus effectively infinite focal length. When the OECD is 'on', the active regions of the FZP diffract a portion of the incident light onto a focal spot. The focal length of the FZP lens is a function of the spacing between the concentric circles in the pattern. b) Cross-section of an OECD. The device consists of a patterned EC layer and gel electrolyte sandwiched between two ITO-coated glass substrates. When a positive voltage is applied to the ITO counter electrode relative to the ITO working electrode, the cations in the gel electrolyte are driven into the EC active PEDOT:PSS patterned regions of the device. When the PEDOT:PSS pattern is electrochemically reduced in response to the applied voltage, a darkening of the patterned layer is observed due to the absorption spectrum shifting into the visible wavelengths. 


\section{Materials and Methods}

In PEDOT:PSS, the electrochemical ion exchange described below changes both the conductivity and the absorption spectrum of PEDOT [10,13,39]:

$$
\text { PEDOT }{ }^{+}: \mathrm{PSS}^{-}+\mathrm{M}^{+}+e^{-} \rightarrow \mathrm{PEDOT}^{0}+\mathrm{M}^{+}: \mathrm{PSS}^{-}
$$

where $\mathrm{M}^{+}$is a cation from the electrolyte, such as $\mathrm{Na}^{+}$or $\mathrm{Li}^{+}$. In the ion exchange, $\mathrm{PSS}^{-}$anions are displaced from their ionic bond with doped $\mathrm{PEDOT}^{+}$, resulting in a dedoped (reduced) $\mathrm{PEDOT}^{0}$ and low conductivity of the film. Due to this electrochemical redox reaction, polaron states within the electronic band structure of the semiconducting polymer are removed, increasing the band gap of PEDOT and making the material resonant with higher energy photons, increasing its absorption at visible wavelengths. This color change is exploited by a typical EC display or window which is a sandwiched structure consisting of a transparent electrode such as indium tin oxide (ITO) on glass or PET film, the EC material, an electrolyte and/or ion storage layer, and a second transparent or metallic counter electrode.

A schematic of a switchable FZP lens and a cross-section of an OECD are shown in Figure 1. The spacing of the concentric ring structure in a FZP decreases as $1 / r^{2}$ where $r$ is the radial distance from the center of the lens. The alternatingly electrochemically active regions with switchable absorption and the chemically over-oxidized inactive regions diffract light at an angle inversely proportional to the ring spacing such that the incident light is diffracted to a single focal point. In binary FZPs, some of the light is diffracted into higher order modes as well, resulting in multiple foci. The power in higher order foci falls off proportional to $m^{-2}$, where $m=1,3,5 \ldots$ is the mode order. To demonstrate proof-of-concept devices, the patterns for the binary FZP lenses in this paper were generated for lens powers of 1.5 and 3.0 diopters (focal lengths of 666 and $333 \mathrm{~mm}$ ) [40]. Each lens has a diameter of $8 \mathrm{~mm}$ and was designed for an incident wavelength of $594 \mathrm{~nm}$. The center circle was designed to be transparent while the spacing between the outermost rings is $25 \mu \mathrm{m}$ and $50 \mu \mathrm{m}$ for the 3.0 and 1.5 diopter lenses, respectively. FZP lenses of higher lens power (shorter focal length) can trivially be fabricated by altering the concentric ring spacing within the constraints of optical lithography. Additionally, linear diffraction gratings were fabricated as patterned lines of alternating inactive and active electrochromic regions. The gratings were designed with pitches of $10,20,30$, and $40 \mu \mathrm{m}$, and a nominal duty cycle of $50 \%$, defined as the ratio of the width of the active lines to the grating pitch.

\section{Device fabrication}

To fabricate the switchable OECD, ITO-coated $25 \mathrm{~mm} \times 25 \mathrm{~mm}$ glass slides (Sigma-Aldrich) with a resistivity

of $8-12 \Omega /$ sq. were used as substrates. The coated slides were cleaned in soap solution, acetone, and isopropanol in a sonicator (Branson) and subsequently dried by blowing with $\mathrm{N}_{2}$ gas. The EC solution consisted of PEDOT:PSS (Clevios PH-1000, Heraeus), $5 \mathrm{v} / \mathrm{v} \%$ glycerol, $0.5 \mathrm{v} / \mathrm{v} \%$ dodecylbenzenesulfonic acid (DBSA, Sigma-Aldrich), and $1 \mathrm{w} / \mathrm{w} \%$ (3-glycidyloxypropyl)trimethoxysilane (GOPS, Sigma-Aldrich) and was spincoated onto the substrate at 1,000 rpm for 60 seconds. The presence of glycerol enhances the conductivity of the PEDOT:PSS formulation while the DBSA serves as a surfactant [41]. GOPS enhances the hydrolytic stability of the formulation and prevent delamination from the underlying substrate [42-44]. PEDOT:PSS was also spincoated onto a blank glass microscope slide as a control in the absence of the ITO layer to measure the thickness and conductivity of the film. After spinning on the PEDOT:PSS solution, the device was baked on a hotplate at $120^{\circ} \mathrm{C}$ for 20 minutes to dry the film. A positive photoresist (AZ 4210, Futurrex) was spin-coated onto the PEDOT:PSS-coated ITO slide and patterned in a Karl Suss MJB3 mask aligner using a photomask with the patterns described above. The photoresist was developed according to the manufacturer's directions and the patterned device was exposed to a $150 \mathrm{~W} \mathrm{O}_{2}$ plasma for 30 seconds to descum the surface. The patterned device was subsequently soaked in a 1:4 solution of commercial bleach (Clorox ${ }^{\mathrm{TM}}, 8.25 \%$ sodium hypochlorite) and water for 5 seconds and then rinsed in DI water. This soak chemically over-oxidizes the PEDOT:PSS and turns the exposed regions into a non-conductive, transparent, and electrochemically inactive film [45]. The photoresist was removed with acetone and methanol and dried by blowing with $\mathrm{N}_{2}$ gas. The true duty cycle of the fabricated gratings was measured from the device micrograph; results are shown in Table 1 . The duty cycle differs from the nominal value due to both over-etching of the photomask and lateral diffusion of the sodium hypochlorite into the masked active regions during the chemical over-oxidization fabrication step 
The final device was assembled as a sandwiched structure composed of a gel electrolyte between the patterned PEDOT:PSS ITO glass slide and a blank ITO-coated glass slide, as shown in Figure 1. Copper tape with conductive adhesive (3M TM Copper Foil Shielding Tape) was wrapped around both the patterned and blank ITOcoated glass slide to make electrical contacts. The gel electrolyte solution was formulated using $40 \mathrm{w} / \mathrm{w} \%$ of poly(sodium 4-styrenesulfonate) (Na:PSS, Sigma-Aldrich) with an average molecular weight of 70,000 Da, 10 w/w \% D-sorbitol, $10 \mathrm{w} / \mathrm{w} \%$ glycerol, and $40 \mathrm{w} / \mathrm{w} \%$ DI water [13]. This viscous formulation was then sandwiched between the patterned PEDOT:PSS ITO glass slide and the blank ITO-coated glass slide with the ITO side facing the electrolyte. A rubber shim with a thickness of 1/32 in. was used as a spacer for the electrolyte. The slides were clipped together and the sandwiched device was then left to dry overnight. Finally, wires were soldered onto the copper tape electrical contacts on the back of the slide for testing.

\section{Device characterization}

The thickness and conductivity of the PEDOT:PSS film on the control glass slide was measured using a Dektak profilometer and four-point probe respectively. The spin-coated PEDOT:PSS film thickness was measured to be $230 \mathrm{~nm}$ with a conductivity of $345 \mathrm{~S} / \mathrm{cm}$. The electrochromic contrast between the patterned regions was measured by viewing the grating device through a transmission microscope with a $594 \mathrm{~nm}$ bandpass filter and comparing the intensity registered by the CMOS camera pixels in the active and inactive (over-oxidized) areas of

the device. The grating contrast $\Delta T$ is defined as the difference in optical transmission between the active and inactive regions and was measured for each grating size at a voltage $0 \mathrm{~V}$ ('off' state, low absorption) and $1 \mathrm{~V}$ ('on' state, high absorption), as show in Table 1. There is a small but measureable diffraction even when the device is off due to a small contrast between the active and inactive regions introduced from the chemical over-oxidation.

As shown in Table 1, the measured contrast in the 'on' state depends on the grating pitch, decreasing significantly for the fine pitch gratings. This can be attributed to lateral diffusion during the chemical over-oxidation patterning step, which is more severe for narrower gratings. Despite this lateral diffusion into the masked regions, the interface between the active and inactive regions is still sharp, so the assumption of a binary diffractive grating is valid (see Figure S1).

The absorption spectrum of the PEDOT:PSS EC layer was measured on an unpatterned PEDOT:PSS coated ITO-glass slide with a spectrophotometer (Ocean Optics ${ }^{\mathrm{TM}}$ ), and is shown in Figure 2. The peak of the absorption spectrum was observed to be between 650-700 nm when the PEDOT:PSS was in the reduced state at a voltage of $1.0 \mathrm{~V}$. At the measurement wavelength of $594 \mathrm{~nm}$, the change in optical density is 0.125 , which

corresponds to a transmission change of $\Delta T=25 \%$ between the 'on' and 'off' states. This measured change in transmission from the spectrophotometer agrees with the measured change between the active and inactive regions observed from the pixel-intensity of the grating micrograph. 


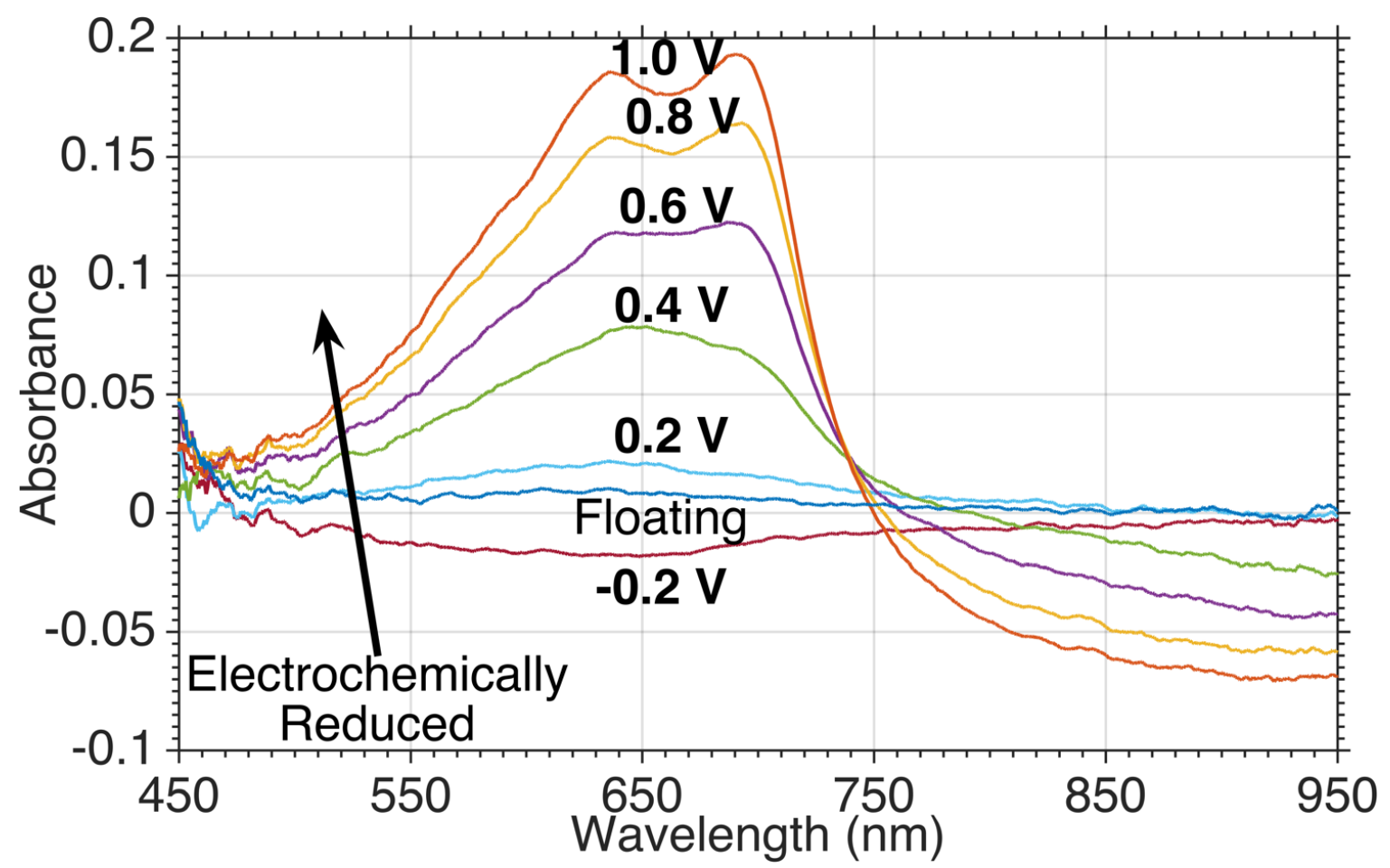

Figure 2: The absorption spectra of unpatterned PEDOT:PSS film in an OECD structure for various applied voltages. The baseline spectrum was measured while the device was floating (open circuit) to account for the absorption in the electrolyte. When reduced ('on' state) the absorption of the film in the visible wavelengths is increased (centered at 650 $\mathrm{nm})$, while the absorption in the infrared wavelengths is decreased (>750 nm)

The diffraction efficiency (DE) of the OECDs was measured using the amplitude diffraction gratings and the optical setup described in Figure 3a. The FZP lens DE and spot size were also characterized using the setup shown in Figure $3 \mathrm{~b}$. The grating DE is defined as the ratio of the optical power in the first-order diffracted spot in the 'on' or 'off' state to the power of the total transmission through the device in the fully oxidized state at -1 V. Referencing the measurement to the fully oxidized state accounts for the optical losses in the electrolyte and at the glass interfaces in order to better estimate the intrinsic OECD DE. The theoretical first-order mode DE of a binary grating is [46]:

$$
\begin{aligned}
& \eta_{m= \pm 1}=\left[\frac{1}{2 \pi}[1-\cos (2 \pi D)] \Delta T\right]^{2} \\
& \eta_{m>1}=\frac{1}{m^{2}} \eta_{m= \pm 1}
\end{aligned}
$$

where $\Delta T$ is the grating contrast, defined as the difference in transmission between the active and inactive EC

regions, and $D$ is the duty cycle of the grating. The DE of the first-order mode in a binary grating is also the DE of the first-order focus in a FZP lens, and each higher order grating diffraction corresponds to a higher order foci in the FZP lens. Figure 4 shows a contour plot of the possible grating DE for different contrasts and duty cycles calculated from equation 1 , with data points to indicate the location of the fabricated gratings within the parameter space. 
Table 1: Diffraction grating performance. The 'On' state is measured at $\mathrm{V}_{\mathrm{L}}=1 \mathrm{~V}$ and the 'Off' state at $\mathrm{V}_{\mathrm{L}}=0 \mathrm{~V}$. The grating contrast is the difference in transmission between the active and inactive regions.

\begin{tabular}{|c|c|c|c|c|c|}
\hline Pitch & $\begin{array}{c}\text { Diffraction } \\
\text { Efficiency, } \\
\text { 'On' }\end{array}$ & $\begin{array}{c}\text { Diffraction } \\
\text { Efficiency, 'Off' }\end{array}$ & $\begin{array}{c}\text { Grating } \\
\text { Contrast 'On' }\end{array}$ & $\begin{array}{c}\text { Grating } \\
\text { Contrast, 'Off' }\end{array}$ & Duty Cycle \\
\hline $\begin{array}{r}10 \\
\mu \mathrm{m}\end{array}$ & $0.037 \%$ & $0.014 \%$ & $6.50 \%$ & $1.48 \%$ & $48.1 \%$ \\
\hline $\begin{array}{r}20 \\
\mu \mathrm{m}\end{array}$ & $0.17 \%$ & $0.059 \%$ & $18.5 \%$ & $1.59 \%$ & $38.2 \%$ \\
\hline $\begin{array}{l}30 \\
\mu \mathrm{m}\end{array}$ & $0.40 \%$ & $0.14 \%$ & $20.0 \%$ & $2.04 \%$ & $44.3 \%$ \\
\hline $\begin{array}{l}40 \\
\mu \mathrm{m}\end{array}$ & $0.52 \%$ & $0.18 \%$ & $24.8 \%$ & $1.55 \%$ & $45.5 \%$ \\
\hline
\end{tabular}




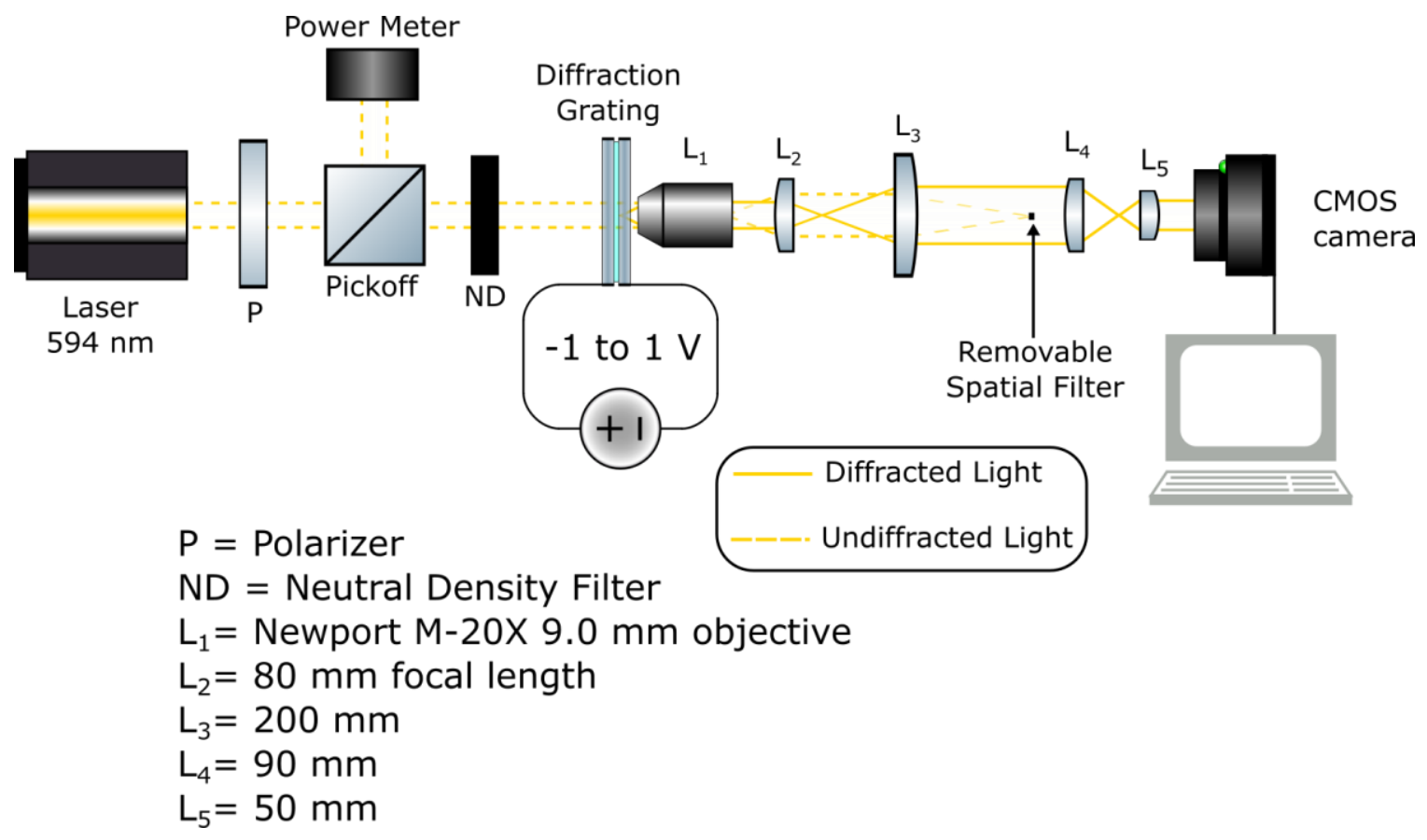

(a)

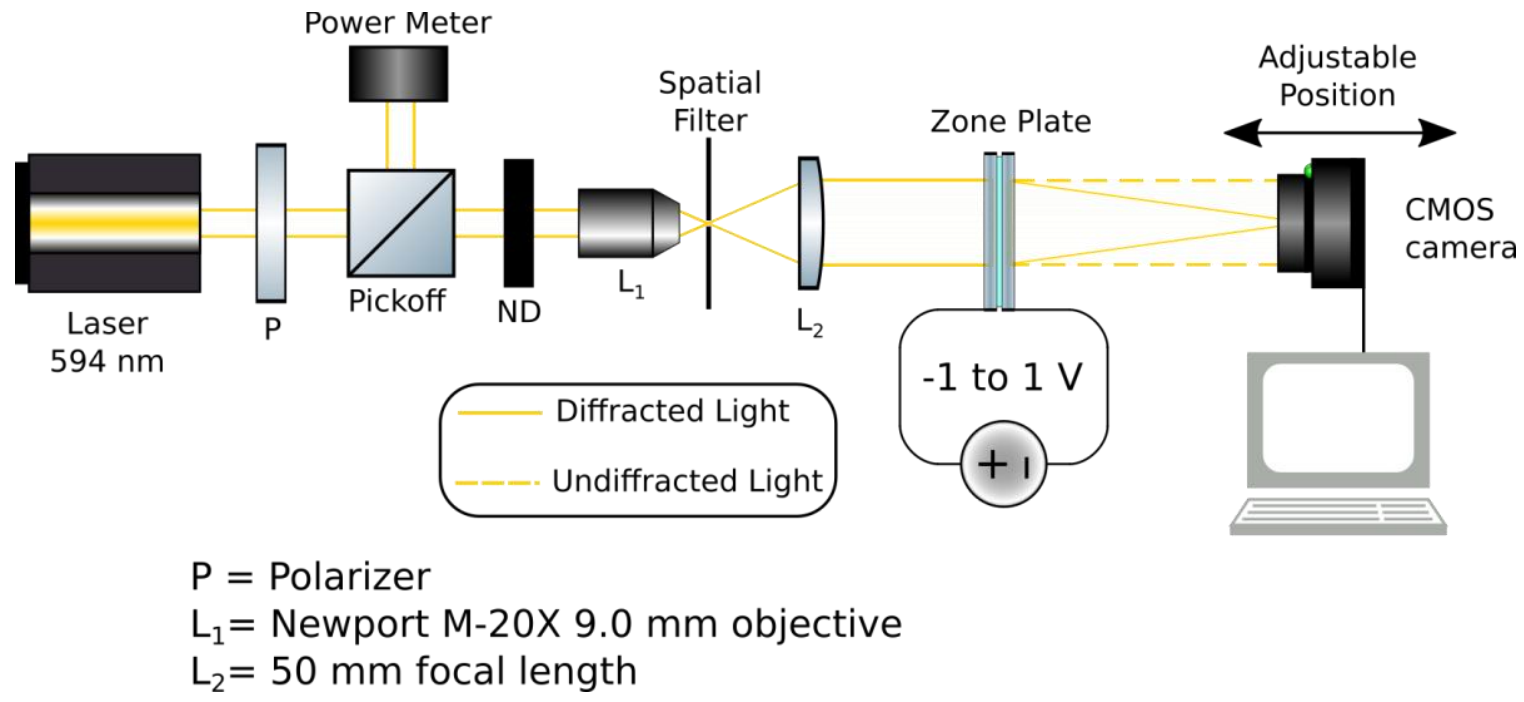

(b) 
Figure 3: Schematic of the optical setup to measure the diffraction efficiency of the OECDs. The $\lambda=594 \mathrm{~nm}$ laser beam is linearly polarized and power fluctuations are monitored with the pickoff mirror and power meter. The removable neutral density filter is used to prevent saturation of the CMOS camera and allows for high dynamic range when measuring the diffraction efficiency.

(a) Experimental setup for measuring the diffraction efficiency of the electrochromic amplitude gratings. Lens $\mathrm{L}_{1}$ images the Fourier transform of both the diffracted and undiffracted light, which is then expanded and projected onto the spatial filter by the telescope $\left(\mathrm{L}_{2}\right.$ and $\left.\mathrm{L}_{3}\right)$. The removable spatial filter blocks the $0^{\text {th }}$ order light, allowing the remaining diffracted orders to be reimaged onto the CMOS camera for measurement. Removal of the spatial filter allows for subsequent measurement of the total transmitted light.

(b) Experimental setup for measuring the diffraction efficiency, focal length, and spot size of the Fresnel zone plate lenses. The laser beam is expanded (lenses $\mathrm{L}_{1}$ and $\mathrm{L}_{2}$ ) to fill the back aperture of each FZP and the focused light is recorded by an adjustable rail-mounted CMOS camera.

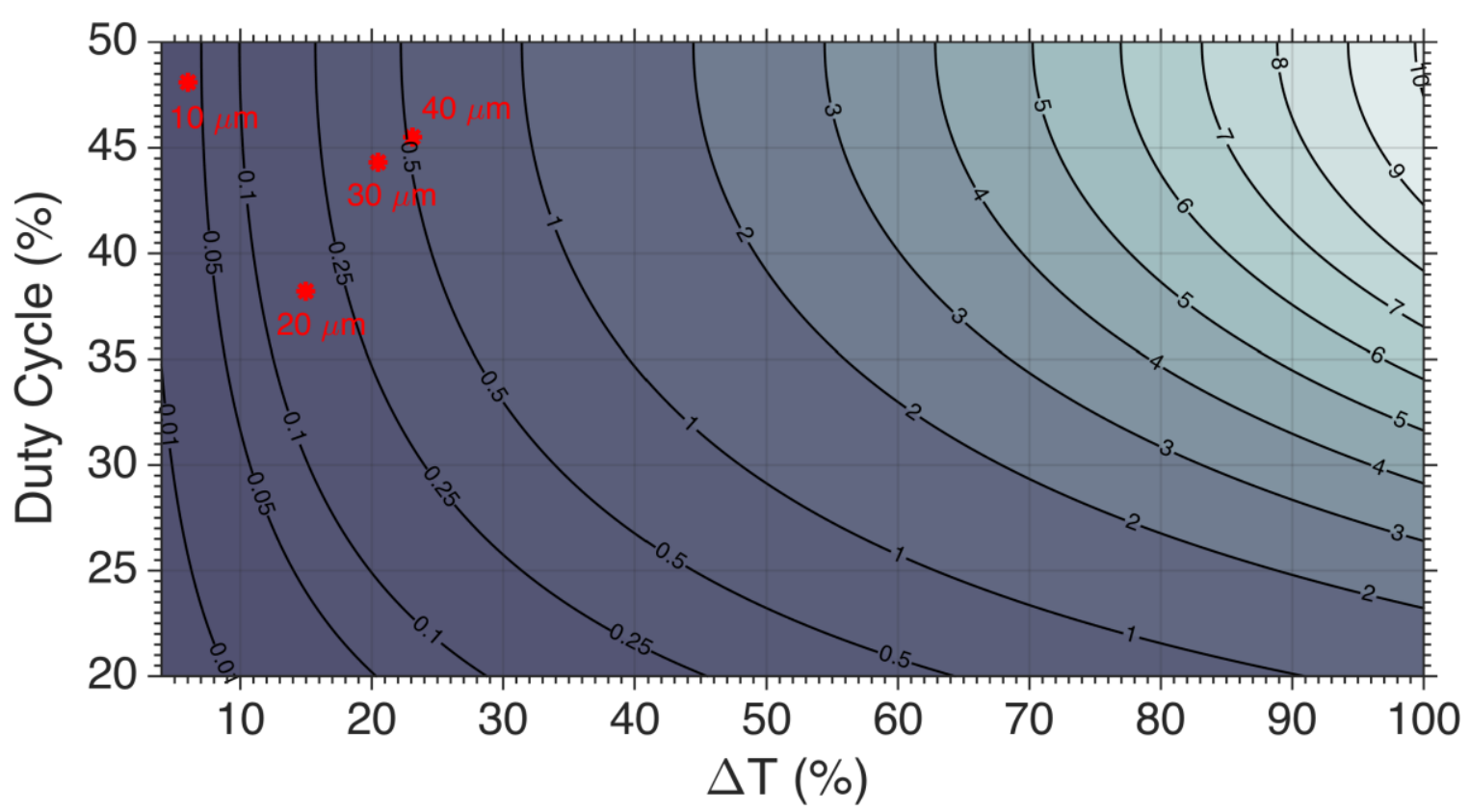

Figure 4: A contour plot of percent diffraction efficiency for the first-order mode in gratings with different grating contrasts and duty cycles. $\Delta \mathrm{T}$ is the difference in transmission between the electrochemically active and inactive patterned regions of the device. The red symbols indicate the fabricated gratings.

\section{Results and Discussion}

\section{Grating Structure}

The measured DE, grating contrast, and duty cycle for the fabricated gratings are shown in Table 1 . The DE of the gratings in the 'off' state is larger than predicted from equation 1 using the measured grating contrast $\Delta \mathrm{T}$ and duty cycle. Indeed equation 1 predicts a DE nearly two orders of magnitude less than the measured value in the 'off' state. This difference can be attributed to a phase shift between the inactive and active regions of the grating due to a slight difference in index of refraction and/or thickness caused by the chemical over-oxidization. Assuming that the diffraction when 'off' is entirely due to a binary phase grating, the optical path difference

between the active and inactive regions would be $\frac{1}{47} \lambda$ or approximately a $3.7 \%$ change. The phase is only significant for the low amplitude contrasts in the 'off' state. Predictably, in the 'on' state the grating contrast is increased, which increases the DE by a factor of 2.9 from the 'off' state to an efficiency of $0.52 \%$ for the $40 \mu \mathrm{m}$ 
grating. The efficiency of the narrower pitch gratings is less due to both the reduced duty cycle and grating contrast from lateral diffusion of the bleach. The DE of the gratings in the 'on' state matches the predicted values from equation 1 using the independently measured values of duty cycle and grating contrast (see Figure S2). This indicates that the diffraction in the 'on' state is primarily due to the absorptive electrochromic amplitude grating, as opposed to any phase effects induced by the electrochemical redox of the PEDOT:PSS. The switching characteristics of the grating and lens structures are shown in Figure 5. The 90\%-10\% fall-time of the device when switching from on-to-off is $55.7 \mathrm{~ms}$, which is significantly faster than the $10 \%-90 \%$ rise time of $13.1 \mathrm{~s}$ when switching from off-to-on. However, the off-to-on switching has a much faster initial rise time of $1.47 \mathrm{~s}$ to reach $70 \%$ of the maximum, and a slower rise to the full intensity afterwards. These characteristics are similar to EC displays which are typically faster during bleaching then during colorization. The effect is usually attributed to the increased conductivity of the PEDOT:PSS film during bleaching, which can be several orders of magnitude larger than in the reduced state [47]. In addition, the large difference in switching time can also be attributed to the Na:PSS electrolyte, which has a large asymmetry between the ionic species. The PSS polyanions are large and mostly fixed in the electrolyte compared to the mobile $\mathrm{Na}^{+}$cations. The movement of the cations into the PEDOT:PSS could be retarded by the huge electrostatic forces from the immobile polyanions in the electrolyte. When driven to the 'off' state, the opposite is true and the cations move out rapidly from the PEDOT:PSS due to these same electrostatic forces. The device switching time can be enhanced by using an electrolyte with lower molecular weight species, such as saline or physiological buffer solution. Additionally, having an electrochemically active counter electrode is known to increase both the rise and fall switching times by providing sufficient charge to fully balance the redox reaction in the PEDOT:PSS [47].

The calculated theoretical maximum DE for the first-order diffracted mode is $10.13 \%$ for a grating contrast of $100 \%$ (completely opaque active region to completely transparent over-oxidized regions) and a duty cycle of 50 $\%$ [46]. In this case of maximum efficiency, the total optical power distribution would be $50 \%$ of the incident

light absorbed in the grating, $25 \%$ diffracted into the $m=0$ order, $10.13 \%$ diffracted into each $m= \pm 1$

orders, and a total of $25 \%$ diffracted into all the $|m| \geq 1$ orders combined. The DE can thus be improved by increasing the electrochromic contrast of the PEDOT:PSS with optimal film properties. Transmission contrasts between oxidized and reduced states of PEDOT:PSS of up to $65 \%$ have been demonstrated in optimized electrochromic displays with increased EC layer thickness [13], which would result in a DE of $4.3 \%$ for the first-order mode. Current passive multifocal IOLs, which use a permanently etched Fresnel lens on top of a refractive lens, provide vision correction with optical energy distributions of up to 7:1 between far and near focus powers [31]. An OECD patterned onto a refractive IOL could thus achieve similar ratios between near and far focus correction in the 'on' state, and in the absence of the applied voltage would default to the 'off' state to provide optimal far vision.

To demonstrate that such enhanced diffraction efficiency is possible with the mentioned techniques, we fabricated a $40 \mu \mathrm{m}$ grating using an organic electrolyte of $1.0 \mathrm{M}$ of $\mathrm{LiClO}_{4}$ in propylene carbonate with $20 \mathrm{wt}$. \% PMMA (35 kDa) filler. The counter electrode was also coated with PEDOT:PSS to act as an ion-storage layer. The duty cycle of $28.5 \%$ resulted in a first-order diffraction efficiency of $1.54 \%$ at $2 \mathrm{~V}$. This DE corresponds to a calculated grating contrast of $64.2 \%$, in line with similar values reported in the literature for electrochromic devices with organic electrolytes [48]. The penalty for this higher contrast is an increase in the required voltage. 


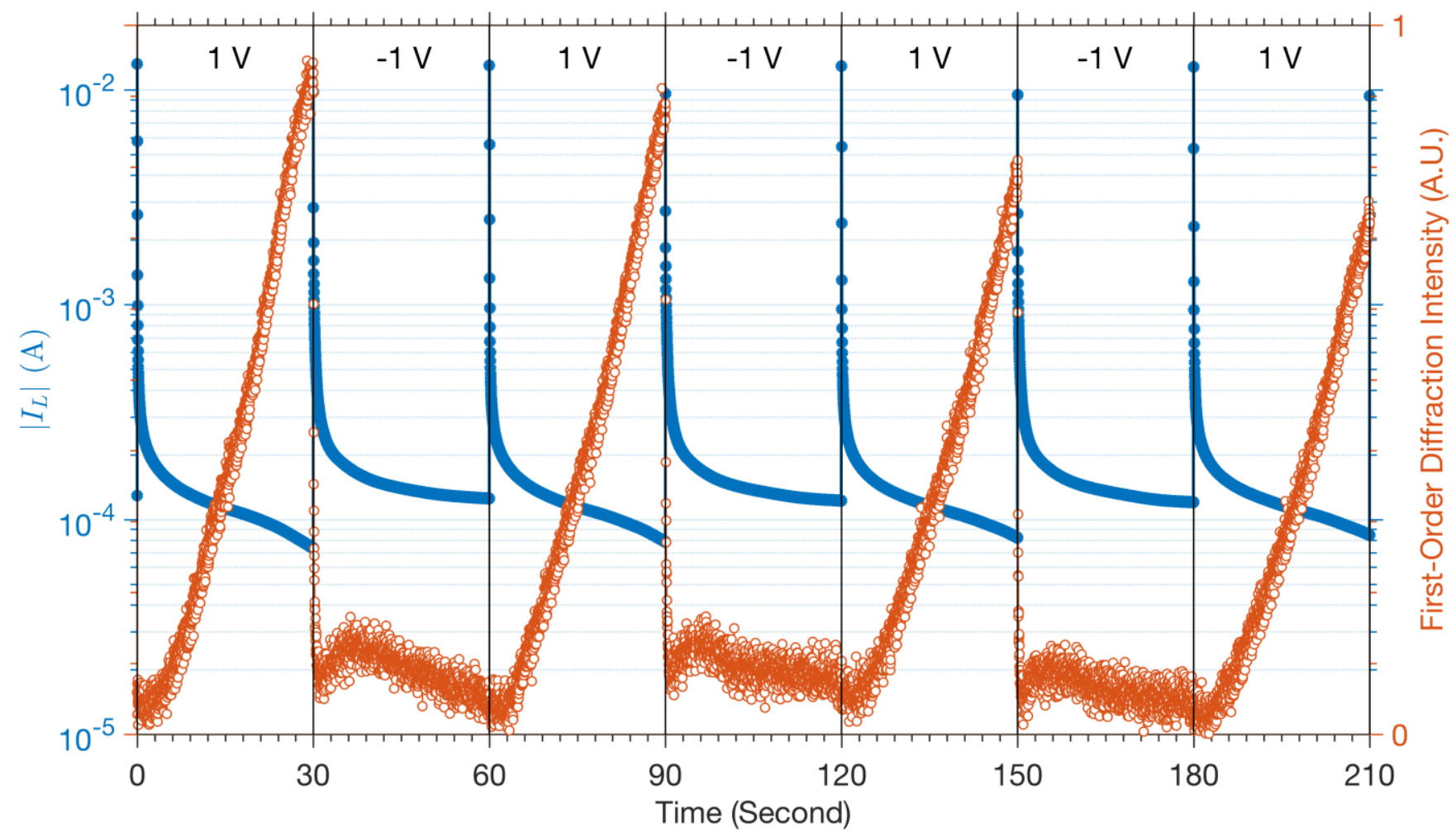

(a)
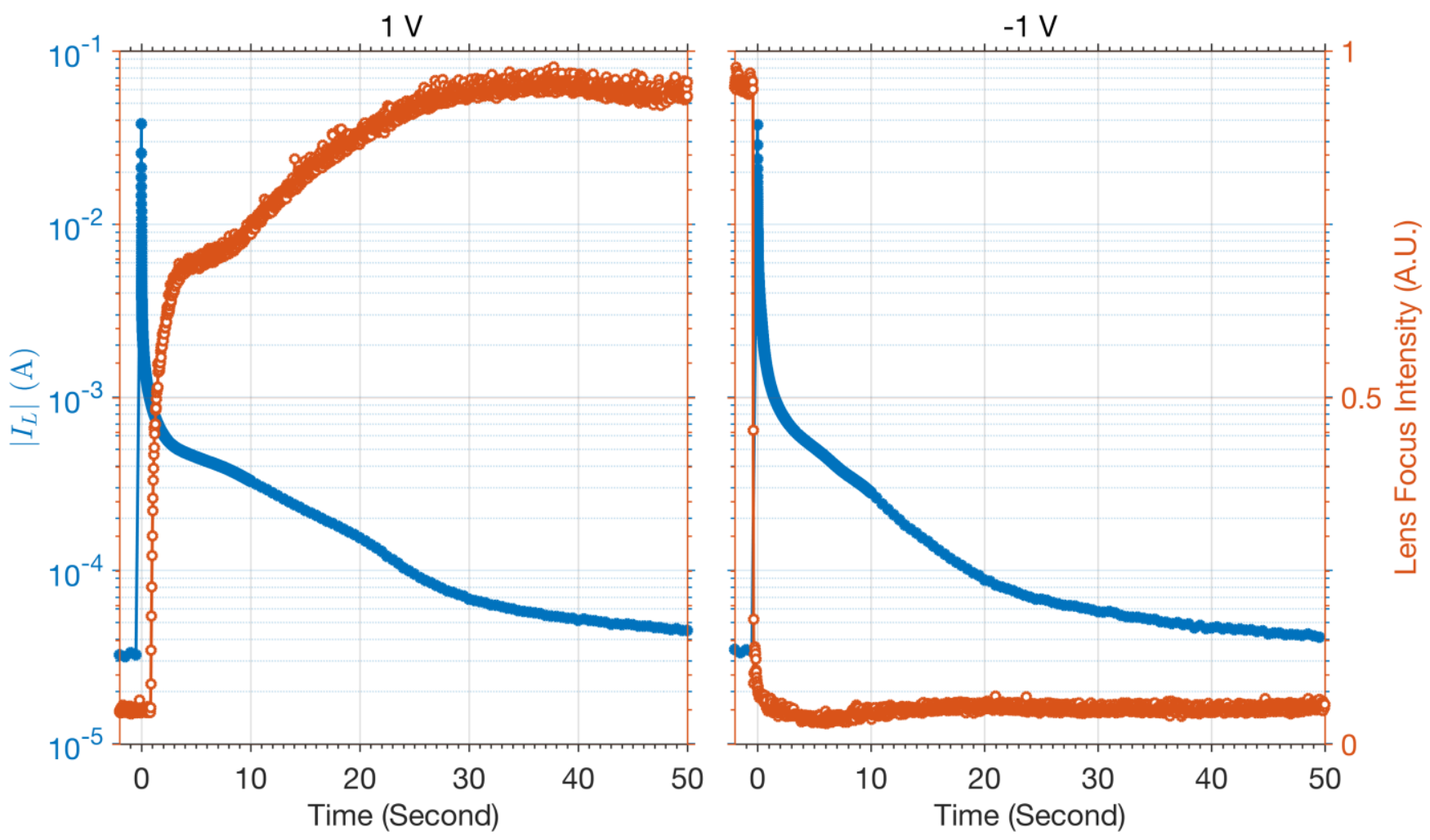

(b)

Figure 5: Switching of OECDs between $-1.0 \mathrm{~V}$ and 1.0 V. a) Diffraction intensity and current over several on/off cycles. The diffraction intensity is the intensity of the $m= \pm 1$ order diffractive spot normalized to the steady-state intensity after 
the device is 'on' for 10 minutes. b) Off-to-on and on-to-off switching transient of the FZP lens.

\section{Fresnel Zone-Plate Structure}

The measured properties of the fabricated OECD FZP lenses are shown in Table 2. The lens focal spot diameter

is the average of the $e^{-2}$ full-width of a 1D Gaussian fit across the X-axis and Y-axis of the focal spot image.

The measured focal spot diameters match well with the theoretical diffraction-limited spot size for a Fresnel lens of $37.6 \mu \mathrm{m}$ and $65.0 \mu \mathrm{m}$ for the $3 \mathrm{D}$ and $1.5 \mathrm{D}$ lens using a radius of $3.0 \mathrm{~mm}$, the same size as the incident beam. The on/off ratios are calculated from the peak of the focal spot in the 'on' state to either the 'off', or the oxidized state. The lens DE was measured from the integrated pixel intensity of the focal spot image to the total integrated intensity into the camera with no lens present. The focal length with the smallest focal spot was found to be at a distance of $34 \mathrm{~cm}$ for the nominally $33.3 \mathrm{~cm}$ (3.0-diopter) lens, and $69 \mathrm{~cm}$ for the $66.6 \mathrm{~cm}$ (1.5-diopter) lens. Figure 6 shows the 1D cross-sections of the 3.0-diopter lens focal spots in different states, as well as a camera image of the focal spots in the 'off' and 'on' states. The switching of the current and optical intensity in the lens focus spot is shown in Figure $5 \mathrm{~b}$ for the FZP lens. This switching time shows the same asymmetry between rise and fall as the grating structure.
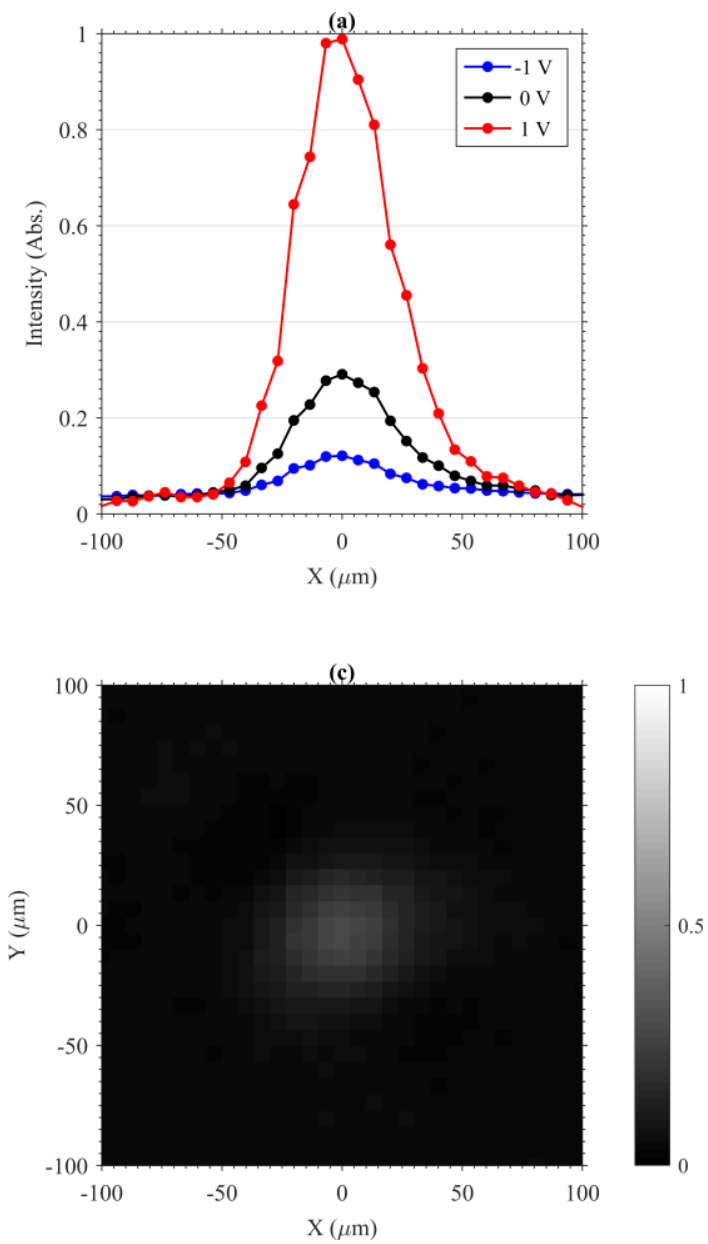
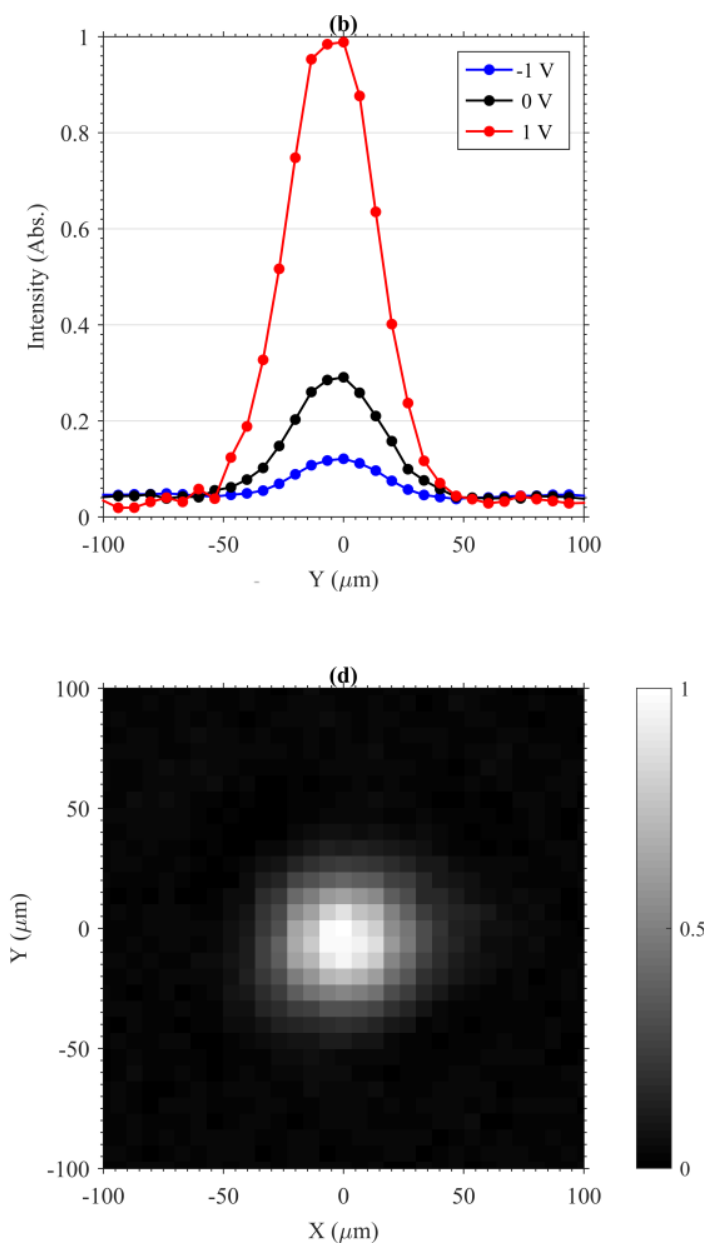

Figure 6: Intensity profile of the focal spot of the FZP OECD. (a) and (b) show the intensity profile of the focal spot of the 3.0 diopter lens in the $\mathrm{X}$-axis and $\mathrm{Y}$-axis, respectively. The ratio of maximum intensity at the focus was 3.4 between $0 \mathrm{~V}$ and $1 \mathrm{~V}$ and 8.2 between $-1 \mathrm{~V}$ and $1 \mathrm{~V}$. (c, d) Image of the lens focus spot at (c) $0 \mathrm{~V}$, 'off' and (d) $+1 \mathrm{~V}$, 'on'. The spot diameter is the average of the $\boldsymbol{B}^{-2}$ full-width of a 1D Gaussian fit across the X-axis and Y-axis of the focal spot and was measured to be $37.6 \mu \mathrm{m}$. The diffraction efficiency in the 'on' state was calculated to be $0.53 \%$.

Table 2: Measured properties of the OECD FZP lenses 


\begin{tabular}{|c|c|c|c|c|c|c|}
\hline $\begin{array}{c}\text { Lens } \\
\text { Power }\end{array}$ & $\begin{array}{l}\text { Spot Size } \\
\text { Diameter }\end{array}$ & $\begin{array}{c}\text { Diffraction } \\
\text { Efficiency } \\
\text { 0 V (Off) }\end{array}$ & $\begin{array}{c}\text { Diffraction } \\
\text { Efficiency } \\
-1 \mathrm{~V} \\
\text { (Oxidized) }\end{array}$ & $\begin{array}{c}\text { Diffraction } \\
\text { Efficiency } \\
1 \text { V (On) }\end{array}$ & $\begin{array}{c}\text { On/Off } \\
\text { Ratio } \\
\text { 0 V to } 1 \mathrm{~V}\end{array}$ & $\begin{array}{c}\text { On/Off } \\
\text { Ratio } \\
-1 \text { V to } 1 \mathrm{~V}\end{array}$ \\
\hline 1.5-Diopter & $68.5 \mu \mathrm{m}$ & $0.35 \%$ & $0.22 \%$ & $0.73 \%$ & 2.6 & 5 \\
\hline 3.0-Diopter & $37.6 \mu \mathrm{m}$ & $0.21 \%$ & $0.13 \%$ & $0.53 \%$ & 3.4 & 8.2 \\
\hline
\end{tabular}

\section{Conclusion}

This paper demonstrates the feasibility of implementing EC materials as active optical devices including gratings, lenses, or other custom diffractive elements, including potential applications in ophthalmic biomedical devices. The difference in peak intensity of the FZP focal spot was shown to vary by a factor of 8.2 and the DE by a factor of 4.1 with a 2 V DC voltage swing. The measured diffraction efficiency matches the calculated efficiency of a binary absorptive diffraction grating using the independently measured transmission grating contrast and duty cycle, indicating that the EC effect is responsible for the measured diffraction. Within ophthalmology, optimized EC-based FZP system can be implemented for specific applications such as varifocal IOLs and contact lenses. This would allow for better control over the focus of post-cataract surgery lens implants, rather than have to use fix multifocal lens with out-of-focus images always present. Optimization of the EC contrast could further improve the potential of OECDs as an optical biomedical device platform.

\section{Acknowledgement}

This work was supported by the National Science Foundation under Grant No. ECCS 1509909 and University of Colorado-Department of Ophthalmology start-up funds.

\section{References}

[1] P.R. Somani, S. Radhakrishnan, Electrochromic materials and devices: Present and future, Mater. Chem. Phys. 77 (2003) 117-133.

[2] R.J. Mortimer, A.L. Dyer, J.R. Reynolds, Electrochromic organic and polymeric materials for display applications, Displays. 27 (2006) 2-18.

[3] C.G. Granqvist, Electrochromism and smart window design, Solid State Ionics. 53-56 (1992) 479-489.

[4] C.G. Granqvist, E. Avendano, A. Azens, Electrochromic coatings and devices: survey of some recent advances, Thin Solid Films. 442 (2003) 201-211. doi:10.1016/S0040-6090(03)00983-0.

[5] C.G. Granqvist, A. Azens, J. Isidorsson, M. Kharrazi, L. Kullman, T. Lindström, et al., Towards the smart window: progress in electrochromics, J. Non. Cryst. Solids. 218 (1997) 273-279.

[6] J.S.E.M. Svensson, C.G. Granqvist, Electrochromic coatings for "smart windows," Sol. Energy Mater. 12 (1985) 391-402.

[7] H.N. Hersh, W.E. Kramer, J.H. McGee, Mechanism of electrochromism in WO3, Appl. Phys. Lett. 27 (1975) 646.

[8] J.S.E.M. Svensson, C.G. Granqvist, Electrochromic coatings for smart windows: Crystalline and amorphous WO3 films, Thin Solid Films. 126 (1985) 31-36.

[9] S.K. Deb, A Novel Electrophotographic System, Appl. Opt. 8 (1969) 192.

[10] Q. Pei, G. Zuccarello, M. Ahlskog, O. Inganäs, Electrochromic and highly stable poly(3,4ethylenedioxythiophene) switches between opaque blue-black and transparent sky blue, Polymer. 35 (1994) 1347-1351. 
[11] R.J. Mortimer, Organic electrochromic materials, Electrochim. Acta. 44 (1999) 2971-2981.

[12] S. Admassie, O. Inganäs, Electrochromism in Diffractive Conducting Polymer Gratings, J. Electrochem. Soc. 151 (2004) H153.

[13] J. Kawahara, P.A. Ersman, I. Engquist, M. Berggren, Improving the color switch contrast in PEDOT:PSS-based electrochromic displays, Org. Electron. 13 (2012) 469-474.

[14] Y.H. Kim, C. Sachse, M.L. Machala, C. May, L. Müller-Meskamp, K. Leo, Highly Conductive PEDOT:PSS Electrode with Optimized Solvent and Thermal Post-Treatment for ITO-Free Organic Solar Cells, Adv. Funct. Mater. 21 (2011) 1076-1081.

[15] B. Winther-Jensen, K. West, Stability of highly conductive poly-3,4-ethylene-dioxythiophene, React. Funct. Polym. 66 (2006) 479-483.

[16] A.M. Nardes, M. Kemerink, M.M. de Kok, E. Vinken, K. Maturova, R.A.J. Janssen, Conductivity, work function, and environmental stability of PEDOT:PSS thin films treated with sorbitol, Org. Electron. 9 (2008) 727-734.

[17] D. Alemu, H.-Y. Wei, K.-C. Ho, C.-W. Chu, Highly conductive PEDOT:PSS electrode by simple film treatment with methanol for ITO-free polymer solar cells, Energy Environ. Sci. 5 (2012) 9662.

[18] D. Khodagholy, J. Rivnay, M. Sessolo, M. Gurfinkel, P. Leleux, L.H. Jimison, et al., High transconductance organic electrochemical transistors., Nat. Commun. 4 (2013) 2133.

[19] V.K. Thakur, G. Ding, J. Ma, P.S. Lee, X. Lu, Hybrid materials and polymer electrolytes for electrochromic device applications., Adv. Mater. 24 (2012) 4071-96.

[20] R.M. Miriani, M.R. Abidian, D.R. Kipke, Cytotoxic analysis of the conducting polymer PEDOT using myocytes, IEEE Eng. Med. Biol. Soc. Annu. Conf. 2008 (2008) 1841-4.

[21] X. Strakosas, M. Bongo, R.M. Owens, The organic electrochemical transistor for biological applications, J. Appl. Polym. Sci. 132 (2015)

[22] M. Asplund, E. Thaning, J. Lundberg, A.C. Sandberg-Nordqvist, B. Kostyszyn, O. Inganäs, et al., Toxicity evaluation of PEDOT/biomolecular composites intended for neural communication electrodes., Biomed. Mater. 4 (2009) 45009.

[23] A. Jonsson, Z. Song, D. Nilsson, B.A. Meyerson, D.T. Simon, B. Linderoth, et al., Therapy using implanted organic bioelectronics, Sci. Adv. 1 (2015)

[24] A. Williamson, M. Ferro, P. Leleux, E. Ismailova, A. Kaszas, T. Doublet, et al., Localized Neuron Stimulation with Organic Electrochemical Transistors on Delaminating Depth Probes, Adv. Mater. (2015)

[25] J. Rivnay, R.M. Owens, G.G. Malliaras, The Rise of Organic Bioelectronics, Chem. Mater. 26 (2014) $679-685$.

[26] D.T. Simon, S. Kurup, K.C. Larsson, R. Hori, K. Tybrandt, M. Goiny, et al., Organic electronics for precise delivery of neurotransmitters to modulate mammalian sensory function., Nat. Mater. 8 (2009) $742-6$.

[27] T. Bhuvana, B. Kim, X. Yang, H. Shin, E. Kim, Reversible full-color generation with patterned yellow electrochromic polymers., Angew. Chem. Int. Ed. Engl. 52 (2013) 1180-4.

[28] Y. Kim, Y. Kim, S. Kim, E. Kim, Electrochromic diffraction from nanopatterned poly(3hexylthiophene)., ACS Nano. 4 (2010) 5277-84.

[29] R. Dehmel, A. Nicolas, M.R.J. Scherer, U. Steiner, 3D Nanostructured Conjugated Polymers for Optical Applications, Adv. Funct. Mater. (2015)

[30] M.J. Moghimi, J. Fernandes, A. Kanhere, H. Jiang, Micro-Fresnel-Zone-Plate Array on Flexible 
Substrate for Large Field-of-View and Focus Scanning., Sci. Rep. 5 (2015) 15861.

[31] G. Ravalico, F. Parentin, P. Sirotti, F. Baccara, Analysis of light energy distribution by multifocal intraocular lenses through an experimental optical model, J. Cataract Refract. Surg. 24 (1998) 647-652.

[32] J.A. Davison, M.J. Simpson, History and development of the apodized diffractive intraocular lens., J. Cataract Refract. Surg. 32 (2006) 849-58.

[33] G. Li, D.L. Mathine, P. Valley, P. Ayräs, J.N. Haddock, M.S. Giridhar, et al., Switchable electro-optic diffractive lens with high efficiency for ophthalmic applications., Proc. Natl. Acad. Sci. U. S. A. 103 (2006) 6100-4.

[34] Y.-H. Fan, H. Ren, S.-T. Wu, Switchable Fresnel lens using polymer-stabilized liquid crystals, Opt. Express. 11 (2003) 3080.

[35] C.J. Woolverton, E. Gustely, L. Li, O.D. Lavrentovich, Liquid crystal effects on bacterial viability, Liq. Cryst. 32 (2005) 417-423.

[36] K. Svennersten, K.C. Larsson, M. Berggren, A. Richter-Dahlfors, Organic bioelectronics in nanomedicine., Biochim. Biophys. Acta. 1810 (2011) 276-85.

[37] K.C. Larsson, P. Kjäll, A. Richter-Dahlfors, Organic bioelectronics for electronic-to-chemical translation in modulation of neuronal signaling and machine-to-brain interfacing., Biochim. Biophys. Acta. 1830 (2013) 4334-44.

[38] R. Green, M.R. Abidian, Conducting Polymers for Neural Prosthetic and Neural Interface Applications., Adv. Mater. (2015).

[39] J.T. Friedlein, S.E. Shaheen, G.G. Malliaras, R.R. McLeod, Optical Measurements Revealing Nonuniform Hole Mobility in Organic Electrochemical Transistors, Adv. Electron. Mater. (2015)

[40] M.C. Saleh, B.E.A. and Teich, Fundamentals of Photonics, 2nd ed., Wiley, 2007.

[41] S. Zhang, P. Kumar, A.S. Nouas, L. Fontaine, H. Tang, F. Cicoira, Solvent-induced changes in PEDOT:PSS films for organic electrochemical transistors, APL Mater. 3 (2015) 14911.

[42] B. Fan, X. Mei, J. Ouyang, Significant Conductivity Enhancement of Conductive Poly(3,4ethylenedioxythiophene):Poly(styrenesulfonate) Films by Adding Anionic Surfactants into Polymer Solution, Macromolecules. 41 (2008) 5971-5973.

[43] J. Ouyang, "Secondary doping" methods to significantly enhance the conductivity of PEDOT:PSS for its application as transparent electrode of optoelectronic devices, Displays. 34 (2013) 423-436.

[44] S. Zhang, E. Hubis, C. Girard, P. Kumar, J. DeFranco, F. Cicoira, et al., Water stability and orthogonal patterning of flexible micro-electrochemical transistors on plastic, J. Mater. Chem. C. 4 (2016) 13821385 .

[45] A.J. Oostra, K.H.W. van den Bos, P.W.M. Blom, J.J. Michels, Disruption of the electrical conductivity of highly conductive poly(3,4-ethylenedioxythiophene):poly(styrene sulfonate) by hypochlorite., J. Phys. Chem. B. 117 (2013) 10929-35.

[46] R. Magnusson, T.K. Gaylord, Diffraction efficiencies of thin absorption and transmittance gratings, Opt. Commun. 28 (1979) 1-3.

[47] T. Deutschmann, E. Oesterschulze, Integrated electrochromic iris device for low power and space-limited applications, J. Opt. 16 (2014) 75301.

[48] T. Deutschmann, E. Oesterschulze, Micro-structured electrochromic device based on poly(3,4ethylenedioxythiophene), J. Micromechanics Microengineering. 23 (2013) 65032. 


\section{Graphical Abstract}

\section{"Off"}

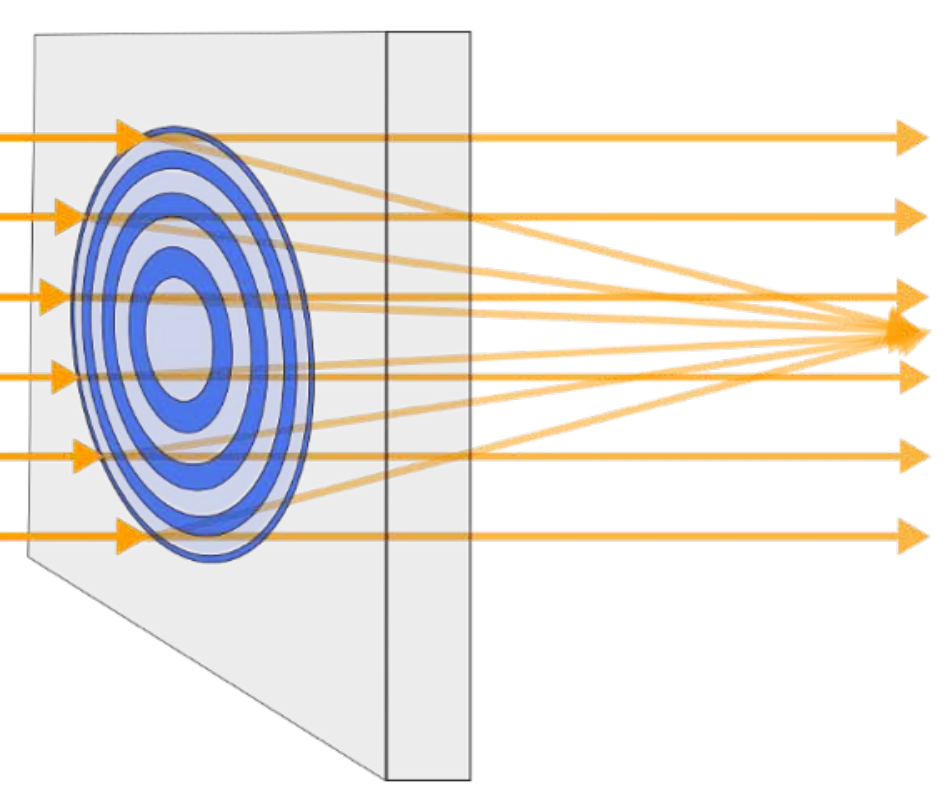

"On"

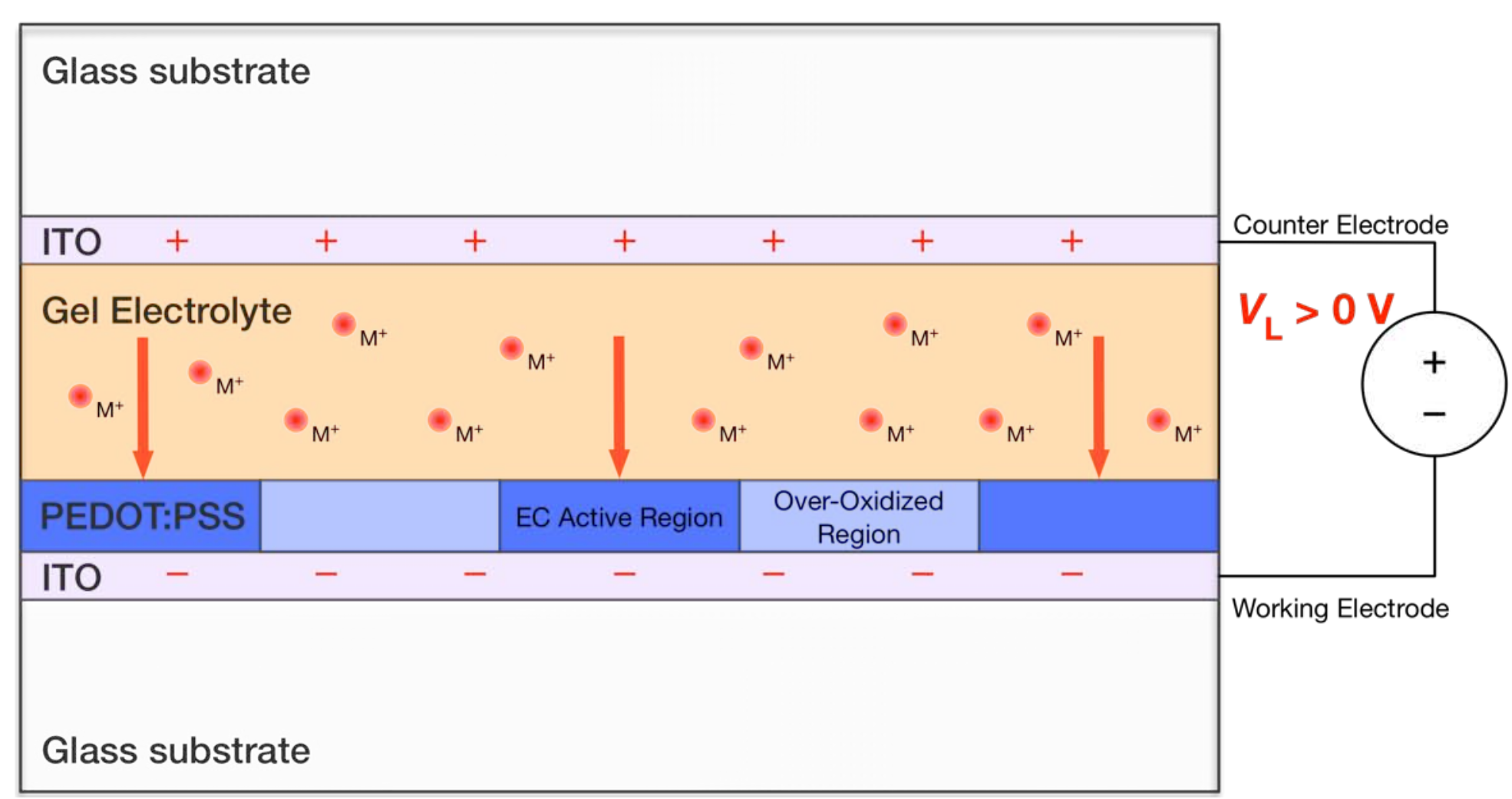

